\title{
Gender-specific influences of weight around conception on glucocorticoid sensitivity in different adipose tissue depots
}

\author{
Petra Bos ${ }^{1}$, Leewen Rattanatray ${ }^{2}$, Severence MacLaughlin ${ }^{1}$, Beverly Muhlhausler ${ }^{1}$, David Gardner ${ }^{3}$, \\ Michael Symonds ${ }^{3}$ and Caroline McMillen ${ }^{1}$ \\ ${ }^{1}$ Sansom Institute, University of South Australia, Adelaide, Australia, ${ }^{2}$ School of Molecular and Biomedical Sciences, \\ University of Adelaide, Adelaide, Australia and ${ }^{3}$ Centre for Reproduction and Early Life, University of Nottingham, \\ Nottingham, UK
}

Numerous studies have shown that maternal diet during pregnancy can have a programming effect on the offspring's risk profile for obesity and metabolic diseases ${ }^{(1,2)}$. One of the factors that can be influenced by prenatal maternal diet is glucocorticoid sensitivity ${ }^{(3)}$, which affects adipogenesis in the offspring. It is not known, however, whether diet around conception influences the offspring's glucocorticoid sensitivity in adipose tissue. The aim was to investigate the effects of periconceptional overnutrition and dietary restriction on expression of genes that influence glucocorticoid sensitivity in different adipose tissue depots of the body.

Twenty-three non-pregnant Merino ewes were included in the study and randomly allocated to one of four treatment groups. The CC group $(n$ 6) received a diet of $100 \%$ metabolisable energy requirements (MER) for 4 months before conception. The CR group $(n$ 6) received a diet of 100\% MER for 3 months followed by 70\% MER for 1 month. The HH group $(n 6)$ was fed ad libitum (170-190\% MER) for 4 months. The HR group ( $n$ 5) was fed ad libitum (170-190\% MER) for 3 months followed by a diet of $70 \%$ MER for 1 month. After 4 months all ewes were artificially inseminated and embryos were transferred into recipient ewes at embryonic day 6-7. Recipient ewes received a diet of $100 \%$ MER at all times. Ewes were allowed to give birth naturally. Lambs $(n 41)$ were humanely killed at 4 months of age; all adipose tissue was collected and weighed at post mortem. Normalised mRNA expression for glucocorticoid receptor (GR) and $11 \beta$-hydroxysteroid dehydrogenase type $1(11 \beta$-HSD-1) was determined in omental, perirenal and subcutaneous adipose tissue using RT-real-time PCR.

Female lambs had higher relative total adipose tissue mass $(P<0.001)$, relative omental $(P=0.002)$, perirenal $(P=0.013)$ and subcutaneous $(P<0.001)$ adipose tissue mass compared with males, independent of treatment group. GR and 11 $\beta$-HSD-1 mRNA expression were not different between genders or treatment groups. GR expression was lower in subcutaneous adipose tissue compared with perirenal $(P<0.001)$ and omental $(P<0.001)$ adipose tissue and lower in omental adipose tissue compared with perirenal adipose tissue $(P=0.022)$. 11ß-HSD-1 expression was not different between adipose tissue depots. Donor-ewe weight around conception was correlated with omental GR $\left(P=0.035, R^{2} 0.223\right)$ and 11 $\beta$-HSD-1 $\left(P=0.043, R^{2} 0.208\right)$ expression in females only. Relative subcutaneous adipose tissue weight was correlated with perirenal 11 $\beta$-HSD-1 expression in females only $\left(P=0.019, R^{2} 0.2688\right)$.

These data show that the expression of GR is different between adipose tissue depots, indicating different functions and mechanisms for glucocorticoid sensitivity in individual depots. Furthermore, these data suggest that different mechanisms are responsible for the regulation of glucocorticoid sensitivity in females and males. These data show that maternal weight around conception influences omental glucocorticoid sensitivity in females but not in males.

1. Godfrey \& Barker (2005) Public Health Nutr 4, 611-624.

2. Symonds ME, Stephenson T, Gardner DS \& Budge H (2007) Reprod Fertil Dev 19, 53-63.

3. Gnanalingham MG, Mostyn A, Symonds ME \& Stephenson T (2005) Am J Physiol Regul Integr Comp Physiol 289, R1407-R1415. 